\title{
High density recording using an ordinary structured MO disk with field modulation
}

\author{
K. Aratani, T. Narahara, A. Fukumoto, and S. Masuhara \\ Advanced Development Laboratories, Sony corporation \\ Kitashinagawa 6-7-35, Shinagawa-ku, Tokyo 141, Japan
}

Abstract - SNR of a polycarbonate MO disk with a narrow track of $0.85 \mu \mathrm{m}$ and with a quadrilayer structure was improved by optimizing and improving the groove geometry, film composition / thickness, and birefringence of the substrate. By using a $690 \mathrm{~nm}$ LD, an NA of 0.55 , magnetic field modulation, and RLL(1,7) coding, a high areal recording density of 2.5 Gbit/in ${ }^{2}$ was obtained. Moreover, the PRML detection of PR $(1,2,1)$ increased the density up to $2.9 \mathrm{Gbit} / \mathrm{in}^{2}$.

KEYWORDS:SNR, Field modulation, Recording density, Polycarbonate, Birefringence, RLL(1,7), PRML

\section{INTRODUCTION}

Hartley and Shannon's equation" (eq.1) describing a channel capacity $C$ in a data transmission channel with a bandwidth $W(\mathrm{~Hz})$ and signal-to-noise ratio SNR is convenient for evaluating $^{23)}$ the recording density of various optical disks regardless of the signal processing applied modulation codes, equalizing, and so on.

$$
C=W \log _{2}(1+S N R) b i t / s
$$

In the case of optical disks, $W$ is proportional to $(\nu / L) \cdot(N A / \lambda)$, where $v$ is a disk scanning velocity and $L$ is the equivalent minimum mark length $\left(L=v /\left(2 f_{\max }\right)\right.$, where $f_{\max }=\operatorname{maximum}$ signal frequency) in units of $\lambda / \mathrm{NA}$, The areal recording density $D$ in units of $(\lambda / N A)^{2}$ is given by dividing $C$ by $\nu$ and by the track pitch $p$ normalized by $N A \lambda . D$ is therefore expressed as

$$
D \propto(I / L p)(N A / \lambda)^{2} \log _{2}(l+S N R)
$$

For an optical disk medium, the quantity of $(1 / L p) \log _{2}(I+S N R)$ is the key for improving $D$.

In general, parameters such as $L, p$, and SNR are not independent of each other. For example, making $l /(L p)$ larger results in a decrease in SNR due to a decrease in CNR or to an increase in crosstalk or W. For an ordinary structured MO disk, improving the SNR at narrow track pitches and finding a good combination of $L, p$, and SNR are required to improve $D$.

The purpose of our study is to estimate the maximum areal recording density $D_{\max }$ obtainable in an ordinary structured MO disk whose SNR has been improved.

\section{EXPERIMENTAL CONDITION}

Quadrilayer structured MO disks as shown in Fig.1 were studied under the experimental conditions listed in Table 1 . The laser wavelength was $690 \mathrm{~nm}$ and the $N A$ of the objective lens was 0.55 . A periodically pulsed laser synchronized with a data clock was used ${ }^{4,5)}$. This writing method has the advantages of writing desired length marks easily and accurately with low writing noise in comparison with the light intensity modulation recording method and the MFM method with a continuously emitting. Although the experimental results were obtained by MFM, these results should be similar to those obtained from light intensity modulation recording as long as the SNR remains unchanged and the techniques used for write-compensation or signal retrieving are sufficiently good. Disk scanning velocity was $4.5 \mathrm{~m} / \mathrm{s}$ for the CNR and crosstalk measurements and was varied from 3.1 to $4.6 \mathrm{~m} / \mathrm{s}$ when changing the bit length. The CNR was measured at $4.3 \mathrm{MHz}$ with a resolution bandwidth of $30 \mathrm{kHz}$ and the crosstalk was measured at $1.6 \mathrm{MHz}$ except when metsuring the CNR and crosstalk dependences on the mark length. 
Table 1. Experimental conditions.

\begin{tabular}{ll}
\hline$\lambda$ & $690 \mathrm{~nm}$ \\
NA & 0.55 \\
Tracking & Continuos \\
Recording area & Groove \\
Read power & $1.2 \mathrm{~mW}$ \\
Magnetic field & $150 \mathrm{Oe}$ \\
Write peak power & $10-14 \mathrm{~mW}$ \\
Write pulse width & $15 \mathrm{~ns}$ \\
\hline
\end{tabular}

\begin{tabular}{ll}
\hline Substrate & $1.2 \mathrm{mmt}$ \\
\hline First Si nitride & $\mathrm{h} 1$ \\
\hline GdFeCo & $5 \mathrm{~nm}$ \\
TbFeCo & $15 \mathrm{~nm}$ \\
\hline Second Si nitride & $\mathrm{h} 3$ \\
\hline Pure Al & $60 \mathrm{~nm}$ \\
\hline Protective layer \\
\hline
\end{tabular}

Fig.1. Layer structure of disk.

\section{MPROVEMENT Of SNR}

As reported previously ${ }^{6}$, an ordinary structured MO disk with high SNR including high CNR and low crosstalk was developed. Let us review the disk optimizing process.

First, the thicknesses of the MO layer, the Si nitride layers, and the Al layer were fixed at $h_{l}=70 \mathrm{~nm}, h_{2}=20 \mathrm{~nm}, h_{3}=25 \mathrm{~nm}$, and $h_{4}=60 \mathrm{~nm}$. The magnetic layer was a $\mathrm{TbFeCo}(15 \mathrm{~nm}) /$ GdFeCo(5nm) exchange-coupled double layer to improve magnetic field sensitivity. The Curie temperature of the TbFeCo layer was $210 \mathrm{C}$ which is high enough to saturate the Kerr rotation at room temperature. Each film thickness was adjusted to maximize the CNR using a glass $2 \mathrm{P}$ substrate even though the CNR was not a sensitive function of the film thickness.

Second, the groove geometry including the track pitch, the groove depth and the groove width were optimized using glass $2 \mathrm{P}$ substrates. Narrowing the track pitch decreases the CNR as shown in Fig.2, which is caused by both a noise increase arising from the groove-land boundary and a carrier level decrease due to the narrower groove width. Narrowing the track pitch also increases crosstalk as shown in Fig.3. These results mean that the
SNR decreases with narrowing pitch. We chose the track pitch of $0.85 \mu \mathrm{m}$ after referring these results as well as to equation (eq.2) to obtain high areal recording density. The groove width was around $0.57 \mu \mathrm{m}$ and the depth was $70 \mathrm{~nm}$. This combination of groove geometry is close to optimum for obtaining $D_{n t a x}$, but is not guaranteed to be the best.

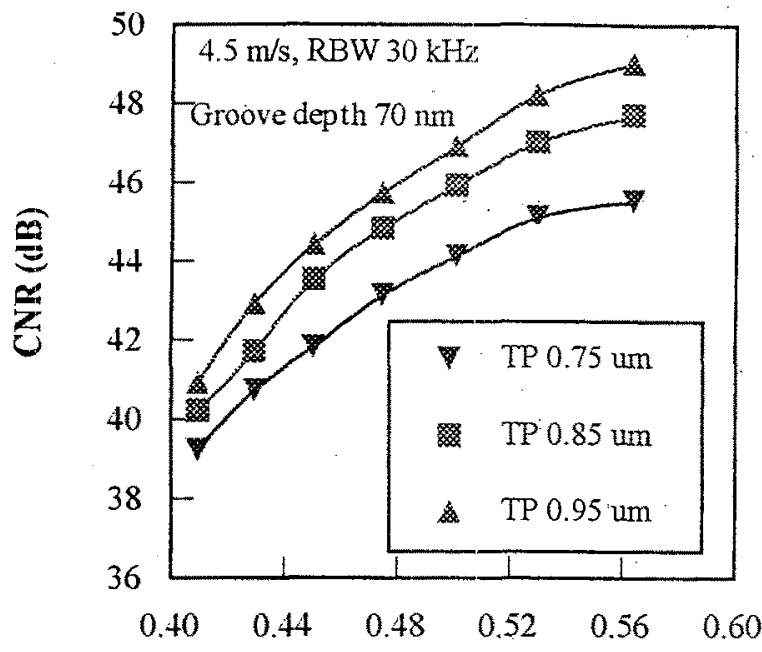

Mark length $(\mu \mathrm{m})$

Fig.2. CNR vs. mark length for various track pitches ${ }^{6)}$.

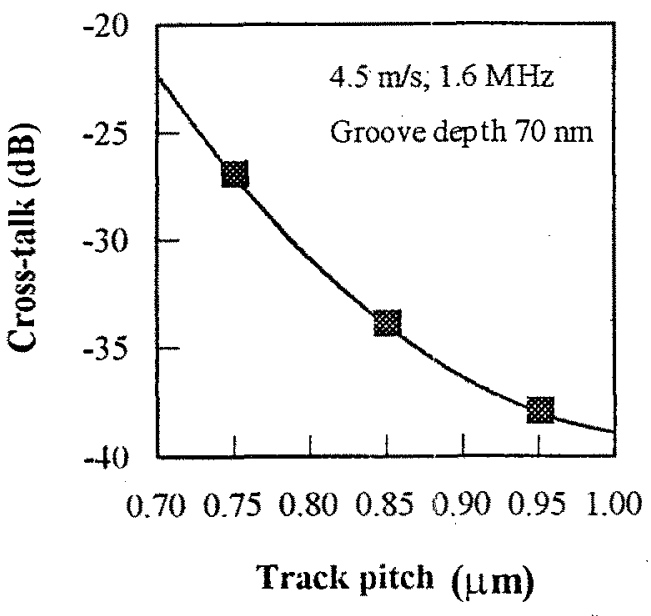

Fig.3. Track pitch dependence of cross-talk ${ }^{(6)}$.

\section{PLASTIC SUBSTRATE}

Injection molded polycarbonate substrates with the same groove geometry as described above were studied. The narrow grooves in the stamper were successfully replicated onto a plastic substrate, which was conlirmed by an SEM and an STM. Two poly-carbonate resins with different opto-elastic coefficicnt resulting in different birefringences were injected. A comparison 
of the two substrates is shown in Table 2, where $d N x z$ is the index difference between the components for the thickness direction and the in-plane direction, which stands for the vertical birefringence.

Table 2 Comparison of polycarbonate substrates.

\begin{tabular}{llll}
\hline & $d N x z$ & CNR & Crosstalk \\
\hline Conventional & $5 \times 10^{-4}$ & $46.3 \mathrm{~dB}$ & $-25 \mathrm{~dB}$ \\
New composition & $2 \times 10^{-4}$ & $46.8 \mathrm{~dB}$ & $-31 \mathrm{~dB}$ \\
Glass 2P & $0 \times 10^{-4}$ & $47.2 \mathrm{~dB}$ & $-33 \mathrm{~dB}$ \\
\hline
\end{tabular}

The birefringence in the in-plane direction was less than $1 \times 10^{-5}$ for both disks. Major differences between the two substrates were found in the crosstalk. A polycarbonate disk made of the new composition shows a CNR and crosstalk comparable to those of a glass substrate.

\section{FINAL TUNING of a DISK}

Due to the birefringence in the substrate or other factors, parameters suitable for a glass disk are not always suitable for the birefringent polycarbonate substrate. Processing conditions such as mastering, injection molding, and sputtering, and parameters including groove geometry and film thickness of the nitride layers $\left(h_{1}\right.$ and $h_{3}$ ) were studied again in order to improve the SNR of the polycarbonate substrate.

The groove width was changed to $0.59 \mu \mathrm{m}$, which contributed a CNR gain of $0.8 \mathrm{~dB}$ for the substrates of both glass and polycarbonate. The change also resulted in an increase in crosstalk by $1.5 \mathrm{~dB}$; the increase, however, was compensated by optimizing $h_{l}$ and $h_{3}$ as described below.

The film thickness dependences of CNR and crosstalk on $h_{t}$ and $h_{3}$ for the polycarbonate substrate are shown in Fig.4 and Fig.5. A disk with $h_{l}=40 \mathrm{~nm}$ shows higher CNR by $1 \mathrm{~dB}$ than that with $h_{l}=70 \mathrm{~nm}$ which was the best thickness for a glass substrate. The thickness $h y$ of the second nitride layer was changed while keeping $h_{3}=40 \mathrm{~nm}$. Both the CNR and the crosstalk were improved by $2 \mathrm{~dB}$ and $4.5 \mathrm{~dB}$, respectively, at $h_{3}$ $=40 \mathrm{~nm}$. Such dependences as shown in Figs. 4 and 5 may be caused by optical complications involving the birefringence, the groove geometry, the Kerr ellipticity, and so on.
From the preceding experiments, the disk based on polycarbonate substrate was finalized with the specifications and characteristics shown in Table 3.

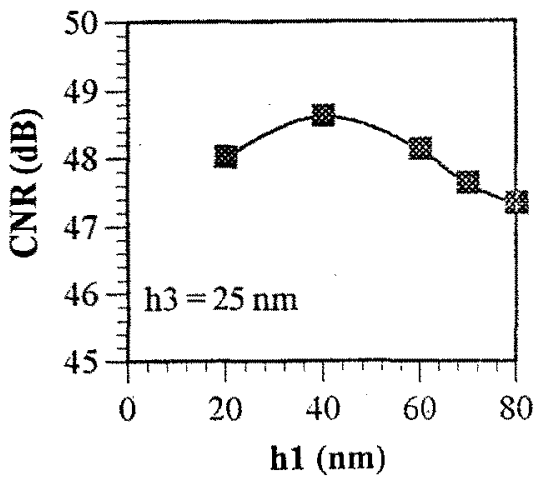

Fig.4 CNR dependence on $h_{1}$.

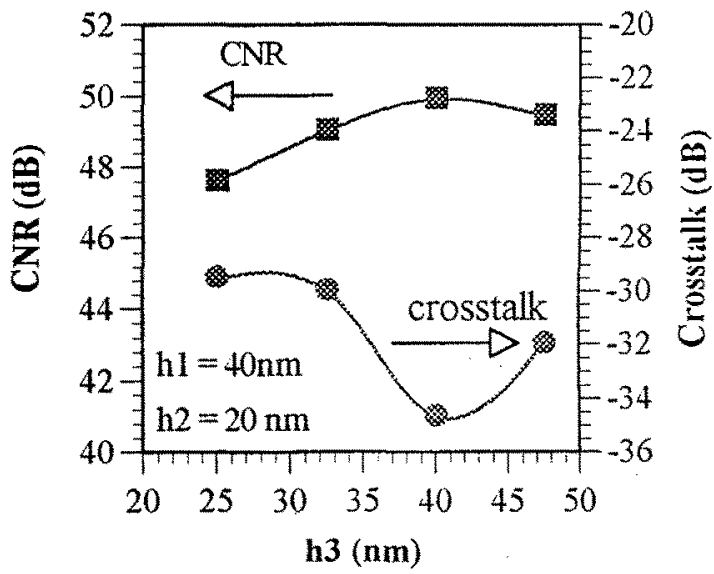

Fig.5 CNR and crosstalk dependence on $h_{3}$.

Table 3. Disk specifications and characteristics.

\begin{tabular}{ll}
\hline$h_{1}$ & $40 \mathrm{~nm}$ \\
$h_{2}$ & $20 \mathrm{~nm}$ \\
$h_{3}$ & $40 \mathrm{~nm}$ \\
Groove width & $0.59 \mu \mathrm{m}$ \\
Groove depth & $70 \mathrm{~nm}$ \\
Track pitch & $0.85 \mu \mathrm{m}$ \\
$d N x z$ & $1.5 \times 10^{-4}$ \\
$d N x y$ & $1.0 \times 10^{-5}$ \\
Reflectivity & 0.17 \\
Kerr rotation & $0.82 \mathrm{deg}$ \\
Kerr ellipticity & $-0.50 \mathrm{deg}$ \\
\hline
\end{tabular}

The mark length dependences of the CNR and crosstalk of the disk are shown in Fig.6. A high CNR of 58.4 dB was obtained at the mark length of $1.9 \mu \mathrm{m}$. The CNR is almost the same as that without the groove noise arising from the groove-land boundary. When changing $h_{3}$ to thinner values, crosstalk was increased not 
only at just $1.6 \mathrm{MHz}$ but for all frequencies.

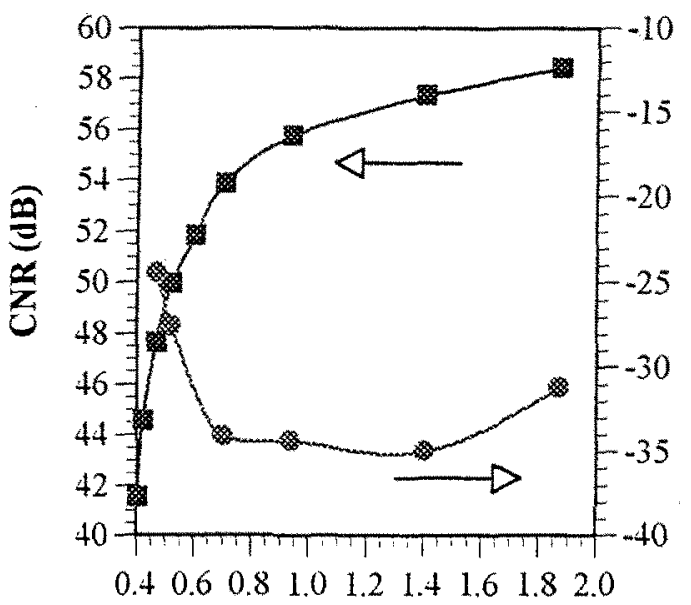

Mark length (ym)

Fig.6. CNR and crosstalk vs. mark length.

\section{LINEAR RECORDING DENSITY}

The linear recording density was studied using two different detection methods. One was the conventional method, and the other was the PRML method of PR $(1,2,1) \mathrm{ML}^{7}$. The modulation code $\mathrm{RLL}(1,7)$ with a channel clock of $18 \mathrm{MHz}$ was used for the both detection methods.

Figure 7 shows the bit error rate vs. the bit length. The minimum error rate was limited to $1.2 \times 10^{-7}$ due to the number of bit measured in our system.

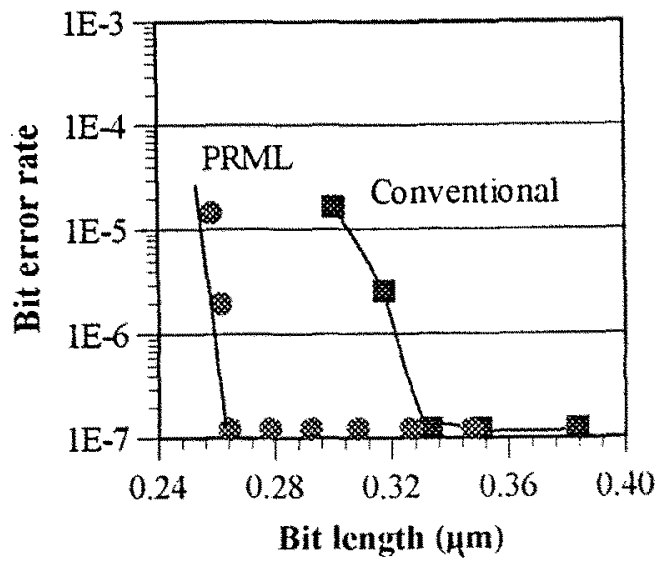

Fig.7. Bit error rate vs, bit length

High linear recording densities of $0.305 \mu \mathrm{m} / \mathrm{bit}$ and $0.260 \mu \mathrm{m} / \mathrm{bit}$ were obtained at an error rate of $1 \times 10^{-5}$ by conventional detection and PRML detection, respectively. These linear recording densities are equivalent to the areal densities $2.5 \mathrm{Gbits} / \mathrm{in}^{2}$ and 2.9 Gbits/in ${ }^{2}$, respectively.

\section{CONCLUSION}

The SNR of an ordinary structured MO disk of a polycarbonate substrate was improved by optimizing and improving the groove geometry, the birefringence in the substrate, and film composition / thickness. The improvements in SNR resulted in the high areal recording density of $2.5 \mathrm{Gbits} / \mathrm{in}^{2}$ by using a $690 \mathrm{~nm} \mathrm{LD}$, and an NA of 0.55 . Moreover, with the help of PRML detection, the density increased to $2.9 \mathrm{Gbits} / \mathrm{in}^{2}$.

\section{ACKNOWLEDGMENT}

We would like to thank N. Arakawa for preparing injectionmolded polycarbonate substrates.

\section{REFERENCE}

(1) C.E.Shannon : Proc. IRE, 37 10(1949).

(2) G.Bouwhuis and J.Braat: Appl.Opt. 17 1993(1978).

(3) S.Kubota and M.Oka : SPIE Proc. 1499 314(1991),

(4) T.Watanabe and H.Ogawa : Proc. Symp. Optical Memory (1988) p.47 [in Japanese].

(5) K.Kataoka, T.Nihara, M.Takahashi and S.Yonezawa: Proc. Symp. Optical Memory (1988)p.53 [in Japanese].

(6) K. Aratani, T. Narahara, A. Fukumoto, S. Masuhara, N. Arakawa, Y. Takemoto and Y. Takeshita : Jap.J.Appl.Phys., 35 (1996) Suppl. 35-1, p433.

(7) T.Narahara, K.Aratani, A.Fukumoto ans S.Masuhara : Proc. Symp. Optical Memory(1996) to be published. 